\title{
Comparison of the Relative Telomere Length Measured in Leukocytes and Eleven Different Human Tissues
}

\author{
D. DLOUHA ${ }^{1}$, J. MALUSKOVA ${ }^{2}$, I. KRALOVA LESNA ${ }^{1}$, V. LANSKA ${ }^{1}$, J. A. HUBACEK ${ }^{1}$ \\ ${ }^{1}$ Center for Experimental Medicine, Institute for Clinical and Experimental Medicine, Prague, \\ Czech Republic, ${ }^{2}$ Clinical and Transplant Pathology Department, Institute for Clinical and \\ Experimental Medicine, Prague, Czech Republic
}

Received June 23, 2014

Accepted July 8, 2014

\section{Summary}

The relative length of telomeres measured in peripheral blood leukocytes is a commonly used system marker for biological aging and can also be used as a biomarker of cardiovascular aging. However, to what extent the telomere length in peripheral leukocytes reflects telomere length in different organ tissues is still unclear. Therefore, we have measured relative telomere length (rTL) in twelve different human tissues (peripheral blood leukocytes, liver, kidney, heart, spleen, brain, skin, triceps, tongue mucosa, intercostal skeletal muscle, subcutaneous fat, and abdominal fat) from twelve cadavers (age range of 29 week of gestation to 88 years old). The highest rTL variability was observed in peripheral leukocytes, and the lowest variability was found in brain. We found a significant linear correlation between leukocyte $r T L$ and both intercostal muscle $(R=0.68, P<0.02)$ and liver $r T L(R=0.60, P<0.05)$ only. High $r T L$ variability was observed between different organs from one individual. Furthermore, we have shown that even slight DNA degradation (modeled by sonication of genomic DNA) leads to false rTL shortening. We conclude that the rTL in peripheral leukocytes is not strongly correlated with the rTL in different organs.

\section{Key words}

Aging • Telomere • Telomere Length • Tissues

\section{Corresponding author}

D. Dlouha, IKEM-CEM-LMG, Videnska 1958/9, 14021 Prague 4, Czech Republic. Fax: +420 241721 666. E-mail: dadl@ikem.cz

\section{Introduction}

Telomeres are situated at the ends of nuclear chromosomes in eukaryotic cells, where they participate in maintaining the integrity and stability of the genome during replication. Telomere length is a reliable indicator of intrinsic biological age and a surrogate for the mitotic clock. Telomeres serve as protective caps, preventing the chromosomal ends from being recognized as double strand breaks by the DNA damage-repair system and the activation of the $\mathrm{p} 53$ or $\mathrm{p} 16^{\mathrm{INK} 4 \mathrm{a}}$ pathway and the start of senescence or apoptosis. Eukaryotic chromosomal telomeres contain short tandem repeat sequences (TTAGGG in humans and other vertebrates). Telomere length is a key determinant of the normal lifespan of a cell and is closely connected with the cell cycle and replicative age (Edo and Andrés 2005). Telomeres that are too short lead to a decrease in functional cells and contribute to overall tissue and organ dysfunction. In the majority of human somatic cells, telomeres decrease in length by 20-200 base pairs during one cell division (Saliques et al. 2010). If the shortening telomeres are not repaired, the shortened telomeres will lead to cell cycle arrest or even apoptosis. An enzymatic complex of several telomerases synthesizes telomeres.

The length of telomeres differs between genders (Butt et al. 2010) and is influenced by the external environment. The most significant factor leading to shortening is oxidative stress (for example, from smoking, alcohol, or UV radiation) (Wong et al. 2010). The length of telomeres between individuals can differ at birth and can be different between people of the same age. The length of telomeres can be somewhat predicated (up to $80 \%$ ) by the length of telomeres of the parents, with the paternal influence on telomere length being 
stronger than that of the maternal influence (Slagboom et al. 1994, Njajou et al. 2007).

The role of telomeres and telomerase in human biology has been studied since the early 1990s because telomere attrition has been implicated in various diseases, including cardiovascular dysfunction, carcinogenesis, and the progression of acute kidney injury (Wills and Schnellmann 2011). Shortened leukocyte telomeres have also been observed in several human diseases (e.g. aplastic anemia, pulmonary fibrosis, and hepatic disease). Some diseases are also associated with mutations in genes encoding telomere-associated proteins (Young 2010).

To determine whether a tissue-specific difference of telomere length exists and if there is a correlation between the rTL found in leukocytes and the rTL found in other tissues, we measured rTL in several different tissues types and leukocytes. Furthermore, we have analyzed the association between DNA integrity and its effects on qPCR measurement of rTL.

\section{Methods}

\section{Samples from cadavers}

We analyzed rTL in twelve different tissues (peripheral blood leukocytes, liver, kidney, heart, spleen, brain, skin, triceps, tongue mucosa, intercostal skeletal muscles, subcutaneous fat, and abdominal fat) from twelve human cadavers.

Twelve anonymous, human cadavers (5 men and 7 women) were examined in our study. The cadavers had no previous history of tumors, metabolic disease (with the exception of diabetes) or systemic inflammation, and no evidence of such diseases was documented during post-mortem pathological examination and autopsy. The routine autopsies were performed less than $12 \mathrm{~h}$ after death. Tissue samples were directly excised from the following parts of the body: heart - margo obtusus sinister; liver - lobus hepatis dexter; kidney - cortex and medula on the level of hilus renalis; brain - lobus frontalis; intercostal skeletal muscles ( $m$. intercostalis externus from 6th intercostal area, $m$. rectus abdominis); skin - abdominal wall; subcutaneous adipose tissue abdominal wall; spleen on the hilus lienalis level; abdominal fat - mesenteric fat; and tongue mucosa dorsum apicis lingue. Due to the inability to remove tissue in some cases, we obtained only 10 skin samples, 7 tongue mucosa samples, and 11 subcutaneous fat samples.
All tissue samples were immediately frozen and stored at $-80^{\circ} \mathrm{C}$ (max for 6 weeks) until further analysis.

\section{Samples from living donors}

We analyzed rTL in peripheral blood leukocytes from nine healthy volunteers of our laboratory ( 3 females, 6 males). Samples were collected in 1988-1990 (age of females was in range of 22-36 years; age of males was in range of 24-50 years) and in 2010 (age of females were in range of 42-56 years; age of males were in range of 44-70 years) from the same donors.

\section{DNA extraction}

Genomic DNA was extracted from tissues $(\sim 10 \mathrm{mg})$ using a QIAmp DNA Micro Kit (Qiagen, Germany). The DNA from living subjects was isolated from leukocytes according the protocol (Miller et al. 1988 ) with slight modifications. All DNA samples were tested for purity and integrity using a Nanodrop 2000 spectrophotometer (Thermo Scientific, USA) and a $1 \%$ agarose gel Electrophoresis System (Bio Rad Power Pac 300).

\section{Preparation of the degraded DNA}

We prepared degraded DNA with fragment sizes between 200 and $300 \mathrm{bp}$ that was subsequently used to test the quality of our qPCR measurements of the rTL in our samples.

We collected one DNA sample from the blood of a living donor, and the DNA was degraded by sonication with a Dr. Hielscher UP 200S, sonotrode S1 (amplitude $70 \%$ and cycle 0.5 ). The DNA solution $(300 \mathrm{ng} / \mu \mathrm{l} ; 600 \mu \mathrm{l})$ was sonicated on ice for up to $5 \mathrm{~min}$ at 30 -s intervals. As ultrasound cannot be defined by physical units, the status of degradation was tested empirically.

\section{Measurement of telomere length}

Telomere length was analyzed as described previously (Dlouha et al. 2012). Analysis was performed using a quantitative polymerase chain reaction (qPCR)-based method with slight modifications (Cawthon 2002). The relative telomere length was calculated as the ratio of telomere repeats to a single-copy gene (SCG) ( $\mathrm{T} / \mathrm{S}$ ratio). The acidic ribosomal phosphoprotein PO (36B4) gene was selected as the SCG. For each sample, the quantity of telomere repeats and the quantity of SCG copies were determined by comparison to a reference sample. The same reference 
DNA was used in all runs to allow for the comparison of results between different runs. All qPCRs were performed in triplicate on the Rotor-Gene 3000 (Corbett Research Ltd). The raw data from each qPCR were analyzed using the comparative quantification analysis software (Rotor-Gene 3000 software, Corbett Research Ltd). The second derivative of the amplification curve was used to identify the peak of the exponential amplification and to determine the Take-Off point of the reaction. The Take-Off point was estimated by finding the first point that was $80 \%$ below the peak level. Based on the Take-Off point and the amplification, our method calculated the relative quantity of telomere repeats or SCG copies in each sample compared to the reference sample. The primers used for the telomere and the SCG amplification were as follows: telomere forward: 5' GGT TTT TGA GGG TGA GGG TGA GGG TGA GGG TGA GGG T, telomere reverse: 5' TCC CGA CTA TCC CTA TCC CTA TCC CTA TCC CTA TCC CTA; SCG forward: 5' CAG CAA GTG GGA AGG TGT AAT CC and SCG reverse: 5' CCC ATT CTA TCA TCA ACG GGT ACA A. The telomere primer concentrations were $135 / 900 \mathrm{nM}$ (forward/reverse, respectively), and the SCG primer concentrations were 300/500 nM (forward/reverse, respectively) (Cawthon 2002, Salpea et al. 2008). In each run, a reference sample, a notemplate control, and all 22 samples were tested in triplicate. The cycling profile for the telomere PCR was as follows: $95{ }^{\circ} \mathrm{C}$ for $10 \mathrm{~min}$, followed by 22 cycles of $95^{\circ} \mathrm{C}$ for $15 \mathrm{~s}$ and $58^{\circ} \mathrm{C}$ for $120 \mathrm{~s}$. For the SCG PCR, the cycling profile was as follows: $95^{\circ} \mathrm{C}$ incubation for $10 \mathrm{~min}$, followed by 35 cycles of $95^{\circ} \mathrm{C}$ for $15 \mathrm{~s}$ and $58{ }^{\circ} \mathrm{C}$ for $60 \mathrm{~s}$. For both the telomere and the SCG PCR, the final reaction volume $(25 \mu \mathrm{l})$ consisted of SensiMix SYBR No-ROX $(2 \times)$ (Bioline Ltd, UK), $30 \mathrm{ng}$ of template and the respective primers. The specificity of all amplifications was determined by melting curve analysis.

To examine the measurement stability of the telomere length by qPCR analysis, both the intra-assay and the inter-assay reproducibility were evaluated. In the intra-assay test, we performed a $\mathrm{T} / \mathrm{S}$ measurement for 8 genomic DNA samples from different tissues from several donors. The samples were simultaneously assayed in heptaplicate repeats in two runs. The intra-assay mean value range of the coefficient of variation from the results of the different DNA sample assays was 1.9-6.9\%. In the inter-assay test, we repeated the $\mathrm{T} / \mathrm{S}$ measurements for the same 8 samples in heptaplicate repeats on the 2 nd and 3rd days. The inter-assay mean value range of the coefficient of variation from the results of the different DNA sample assays was 3.4-14.8\%.

\section{Statistical analysis}

Correlation coefficient was calculated for the respective data sets and its value is plotted in the graphs denoted as R. The lines in graphs represent simple linear regression. We used also nonparametric Spearman's test and $\mathrm{P}<0.05$ value for comparison of the rTL in leukocytes and in different tissues.

\section{Results}

Determination of relative telomere length in different tissues from cadavers

As expected, there was high variability in the relative telomere length between individuals, possibly due to the large age span of the samples analyzed. We found an inverse relationship between rTL and donor age, with the longest rTL detected in the youngest donors (Fig. 1A and Table 1).
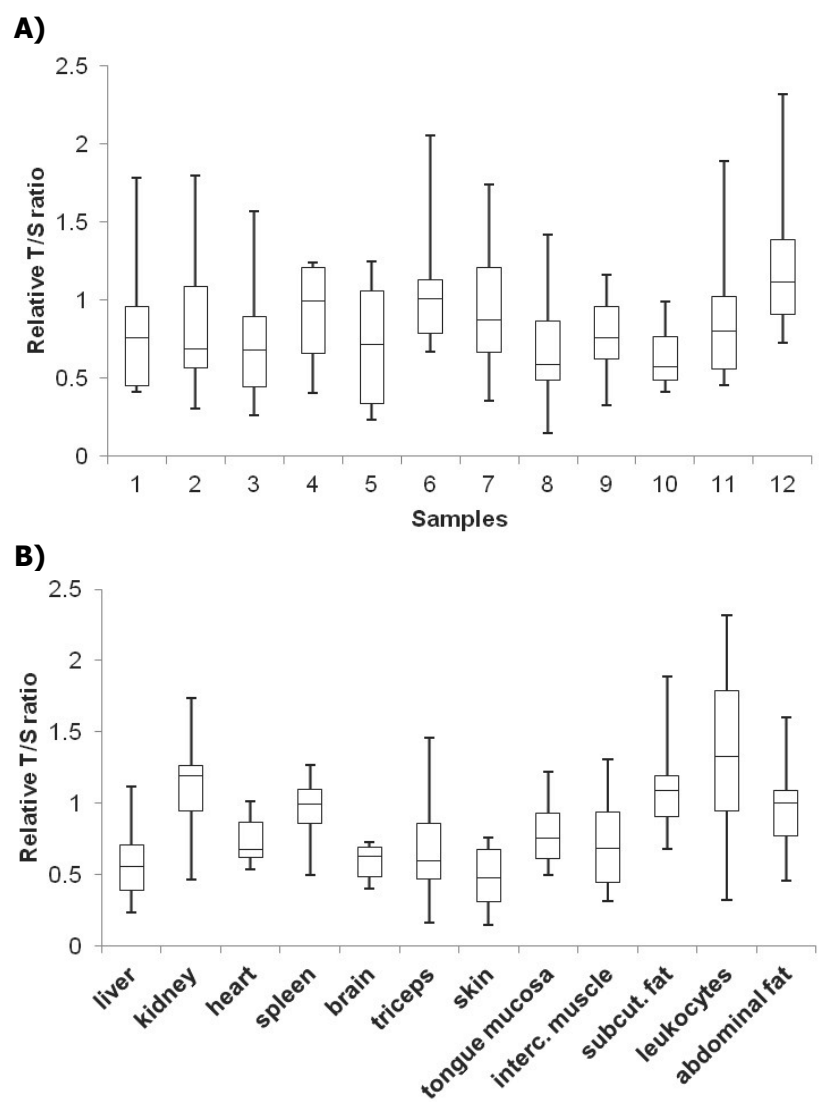

Fig. 1. The range of relative $T / S$ ratio measured in DNA from dead donors according to samples A) and according to tissues B). The relative telomere length is calculated as the ratio of telomere repeats to a single-copy gene $-\mathrm{T} / \mathrm{S}$ ratio. Box Plots express Median: Box: $25 \%$, $75 \%$; Whisker: Non-Outlier Min. Non-Outlier Max. 


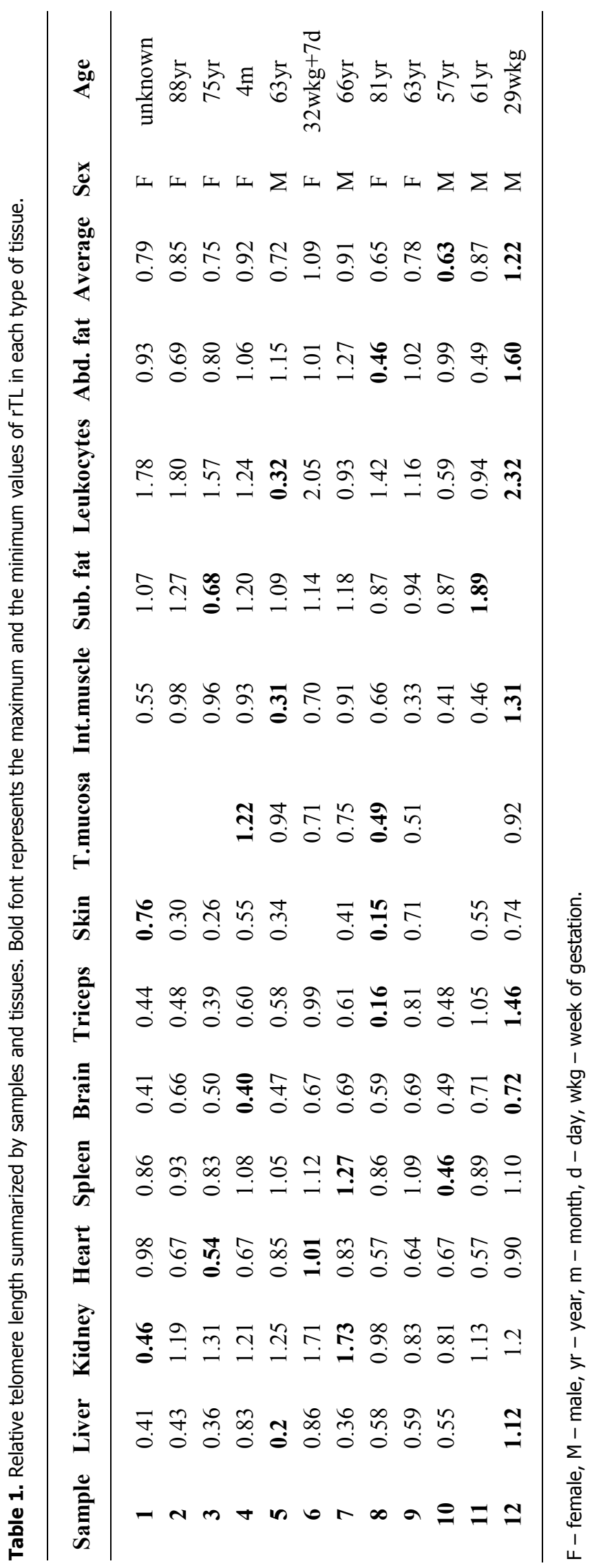


Interestingly, the variability of rTL within organs from the same individual was also very high, with differences reaching upwards of $50 \%$.

The relative telomere length was significantly higher in blood compared to liver, brain $(p<0.001)$, heart, $\mathrm{m}$. triceps, intercostal muscles, skin $(\mathrm{p}<0.01)$, spleen and mucosa $(\mathrm{p}<0.05)$ but was not different compared to adipose and renal tissue. The longest rTL was detected in leukocytes (1.34 times longer than the reference telomere) and kidney (1.15), and the shortest was found in skin (0.48), liver (0.57), and brain (0.58) (Fig. 1B). These data, however, reflected the means of our analysis and were not valid for all individuals. The largest rTL variability was observed in leukocytes and kidney; the smallest rTL variability was observed in brain, heart and skin (Table 1).

Without regard to donor age, we found a significant $(\mathrm{P}<0.05)$ linear correlation between leukocyte rTL and both intercostal muscle rTL $(\mathrm{R}=0.68)$ and liver rTL $(\mathrm{R}=0.60)$. As observed in Figure 2, the rTL in all other organs shows little to no correlation with the rTL in leukocytes. We found a low correlation between the rTL in leukocytes and aortic tissue $(\mathrm{R}=0.33)$. Low or no correlation was detected between the rTL in leukocytes and the rTL in brain tissue $(\mathrm{R}=0.28)$, triceps ( $\mathrm{R}=0.34)$, mucosa $(\mathrm{R}=0.09)$, spleen $(\mathrm{R}=0.22)$, kidney tissue $(\mathrm{R}=0.07)$ or skin $(\mathrm{R}=0.29)$. Additionally, the $\mathrm{rTL}$ in samples from abdominal fat and subcutaneous fat were not correlated with the rTL in leukocytes $(\mathrm{R}=0.13$ and $\mathrm{R}=0.09$, respectively).

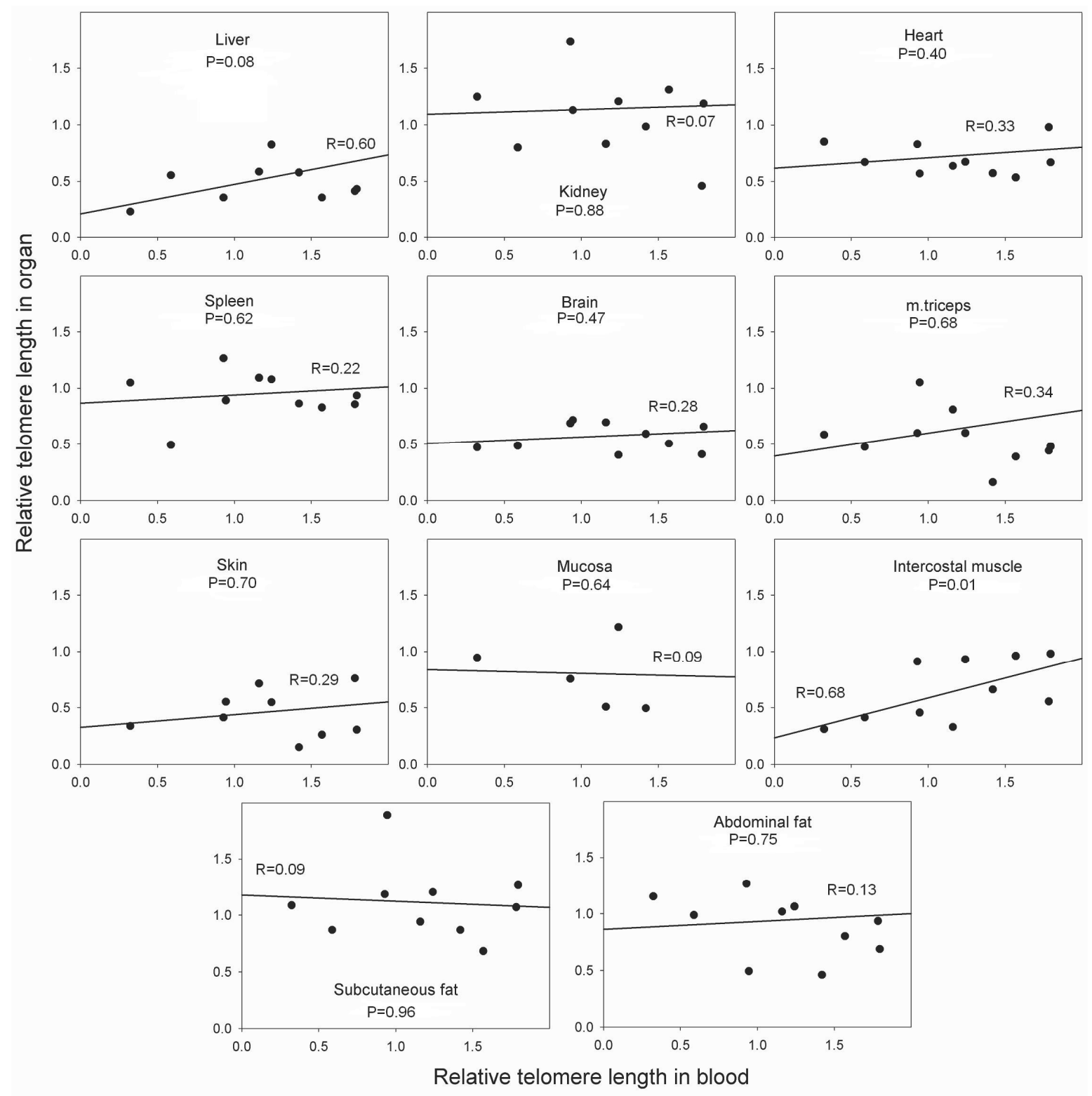

Fig. 2. Correlation between $r T L$ in leukocytes and $r T L$ in different human organs. $R$ - Correlation coeficient, $P-P$ value. 


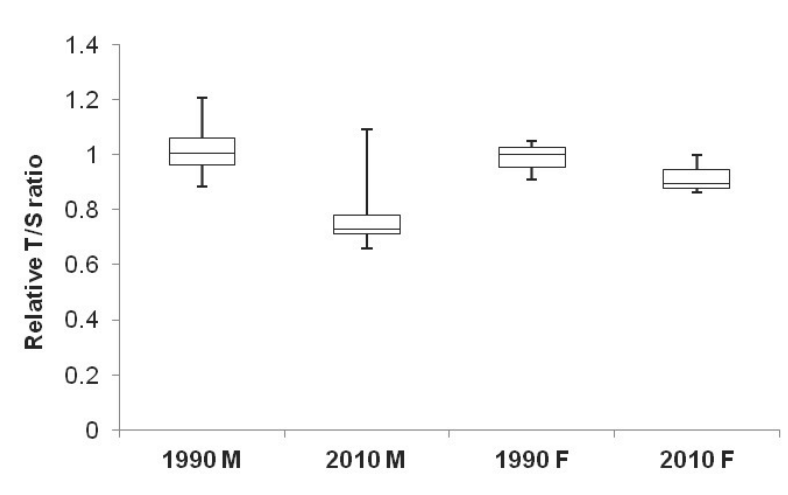

Fig. 3. Comparison of $T L$ in leukocytes from living donors. The steepness of decline is more noticeable in men compare to women which correlates with TL gender differences. F - female, M - male. Box Plots: for Males: Medians $=1.0$ and 0.73; $25 \%-75 \%=(0.96 ; 1.06)$ and $(0.71,0.78)$; Non-Outlier Range $=(0.89,1.21)$ and $(0.66,1.1)$. For Females: Medians $=1.0$ and $0.89 ; 25 \%-75 \%=(0.96 ; 1.02)$ and $(0.88,0.95)$; Non-Outlier Range $=(0.91,1.05)$ and $(0.86,1.0)$.

\section{rTL in living donors}

Our study has confirmed that the rTL decreases in both men and women during aging. In addition, we observed a slightly faster reduction in the rTL in men compared to women. The decline of rTL was in range 0.12-0.32 for men and 0.05-0.11 for women (Fig. 3).

\section{Differences between the rTL from degraded DNA}

We compared the rTL in a degraded DNA sample to that in non-degraded samples. Our experiment showed that rTL decreases with the deteriorating quality of nucleic acids. The rTL decreased in an approximately linearly manner with respect to the integrity of the DNA sample (Fig. 4).

\section{Discussion}

In our study, we focused on the correlation between the relative telomere length (rTL) in leukocytes and the rTL in eleven other human tissues. We found some correlation between the rTL in circulating leukocytes and the rTL in both the intercostal muscles and the liver. All other analyzed tissue did not show a significant relationship with the rTL in leukocytes. We detected a slight trend towards a correlation between the rTL in leukocytes and the rTL in both brain tissue and spleen.

To our knowledge, this study is the first that focused on the analysis of rTL in a variety of human tissues. There are a few studies that have focused on a similar topic, but they focused on a tissue type. A study by Wilson et al. (2008) found some relationship between

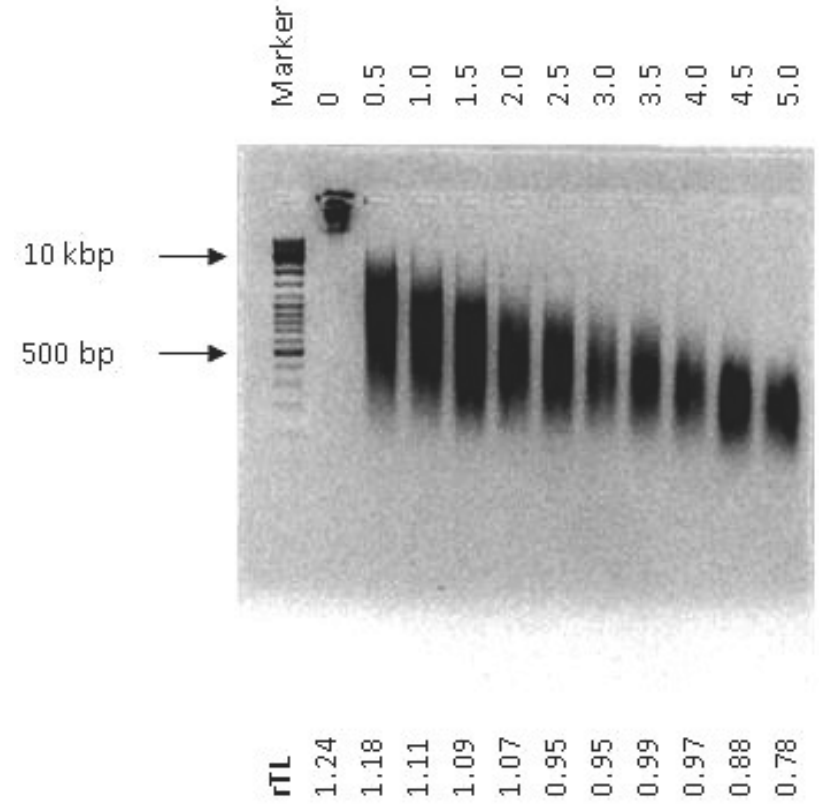

Fig. 4. Degradation of DNA. $180 \mu \mathrm{g}$ DNA were sonicated for up to $5 \mathrm{~min}$, aliquots of $25 \mu \mathrm{l}$ were taken every $30 \mathrm{~s}$ and the DNA was electrophoresed in an ethidium bromide-stained $1.2 \%$ agarose gel. The used concentration of non-degraded and degraded samples was $300 \mathrm{ng} / \mu \mathrm{l}$. A 100 bp ladder (GeneRuler ${ }^{\mathrm{TM}}$ DNA Ladder Mix) was used as size standard. The relative telomere length detected in degraded DNA samples (300 bp) was approximately $1.6 \mathrm{x}$ lower than in non-degraded samples (>10 kbp).

the rTL in leukocytes and the rTL in aortic tissue, but the question still remains if the rTLs are correlated. Some studies (Yang and Fogo 2010, Wills and Schnellmann 2010, 2011) observed a decline in the rTL in different parts of kidney tissue with age. A direct correlation between the rTL in peripheral leukocytes and the rTL in human kidney tissue has not yet been described. In contrast to results (Friedrich et al. 2000) we did not find an association between the rTL in leukocytes and the rTL in skin.

In humans, rTL is extensively measured in leukocytes in relation to aging and various pathologies. Telomere length in leukocytes is highly variable among individuals and decreases throughout the persons' lifespan. Large differences in telomere length develop in the first few years after birth but are relatively stable throughout childhood and the preadolescent and adolescent years (Rufer et al. 1999). Some human disorders associated with shorter telomere length are caused by a defective telomerase function or mutations in the DNA repair system. An important aging hypothesis is that telomere attrition increases at the onset of disease. Therefore, it has been suggested that leukocyte telomere length could be a good marker for disease (Mitchell et al. 
1999, Oeseburg et al. 2010).

The results of our study should be analyzed with regard to the biology of the tissues used. Leukocytes are comprised of different cell lines, and their proportions in the blood are highly variable even in healthy humans. Leukocytes consist mainly of neutrophil granulocytes; the proportion of this cell line ranges from $40-70 \%$, and these cells have a very short lifespan of only 1-3 days. Our study examined telomere length in this major blood cell population. In contrast, most neurons, once differentiated, can live for more than 100 years. Additionally, fibroblasts are typical, non-dividing cells that can survive for many years. The fact that telomere length was significantly higher in blood compared to most of the tissues studied could reflect the expected life span of the different cell types.

A drawback of our study is the fact that many of our samples were from seniors over 57 years of age and infants (two were preterm neonates, and one infant was 4 months old). Unfortunately, we did not have any middle-age donors, as it is quite difficult to obtain these samples. The relatively small number of donors or the different pathologies and diseases that led to the morbidity and mortality of our donors could have also influenced these results. Telomere length can also be regulated by epigenetic factors (García-Cao et al. 2004), which we have not analyzed. The epigenetic regulation of telomere length may also have an impact in cancer pathology and aging. As previously described, rTL is influenced by external environmental factors, with the most significant of these being oxidative stress. The risk factors associated with oxidative stress, such as smoking, UV radiation, and alcohol intake (Wong et al. 2010, Stranberg et al. 2012), may have contributed to the variability of our study, but because we have collected samples anonymously, no such information is available.
Confirming results from previous studies, we found that rTL differs between women and men (Slagboom et al. 1994). In addition, we detected a rapid decline of rTL in middle-age men which correlates with faster aging compared to women. Our study also suggests that the decline in rTL is variable depending on the different stages of human life.

Analyses of the rTL of DNA with deceased integrity and purity confirmed the necessity to use DNA with high purity and $10 \mathrm{kbp}$ in size. The improper storage and treatment of DNA could lead to a distortion of rTL results.

\section{Conclusion}

Despite the relatively low number of individuals analyzed and the large age span of the donors, we suggest that there is a very low correlation between the rTL within most tissues and the rTL in blood leukocytes. Thus, the use of leukocytes as a source of DNA for rTL analysis for the estimation of individual "tissue age" is questionable.

\section{Conflict of Interest}

There is no conflict of interest.

\section{Acknowledgements}

Authors thank Prof. Frantisek Koukolik and his colleagues from the Department of Pathology and Molecular Biology, Thomayer Hospital, Prague, Czech Republic, for providing anonymous tissue samples. Study was supported by Ministry of Health, Czech Republic conceptual development of research organization (,Institute for Clinical and Experimental Medicine IKEM, IN 00023001“).

\section{References}

BUTT HZ, ATTURU G, LONDON NJ, SAYERS RD, BOWN MJ: Telomere length dynamics in vascular disease: a review. Eur J Vasc Endovasc Surg 40: 17-26, 2010.

CAWTHON RM: Telomere measurement by quantitative PCR. Nucleic Acids Res 30: e47, 2002.

DLOUHA D, PITHA J, LANSKA V, HUBACEK JA: Association between FTO 1st intron tagging variant and telomere length in middle aged females. 3PMFs study. Clin Chim Acta 413: 1222-1225, 2012.

EDO MD, ANDRÉS V: Aging, telomeres and atherosclerosis. Cardiovasc Res 66: 213-221, 2005.

FRIEDRICH U, GRIESE E, SCHWAB M, FRITZ P, THON K, KLOTZ U: Telomere length in different tissues of elderly patients. Mech Ageing Dev 119: 89-99, 2000. 
GARCÍA-CAO M, O'SULLIVAN R, PETERS AH, JENUWEIN T, BLASCO MA: Epigenetic regulation of telomere length in mammalian cells by the Suv39h1 and Suv39h2 histone methyltransferases. Nat Genet 36: 94-99, 2004.

MILLER SA, DYKES DD, POLESKY H: A simple salting out procedure for DNA extraction from human nucleated cells. Nucleic Acid Res 16: 1215, 1988.

MITCHELL JR, WOOD E, COLLINS K: A telomerase component is defective in the human disease dyskeratosis congenita. Nature 402: 551-555, 1999.

NJAJOU OT, CAWTHON RM, DAMCOTT CM, WU SH, OTT S, GARANT MJ, BLACKBURN EH, MITCHELL $\mathrm{BD}$, SHULDINER AR, HSUEH WC: Telomere length is paternally inherited and is associated with parental lifespan. Proc Natl Acad Sci USA 104: 12135-12139, 2007.

OESEBURG H, DE BOER RA, VAN GILST WH, VAN DER HARST P: Telomere biology in healthy aging and disease. Pflugers Arch 459: 259-268, 2010.

RUFER N, BRUMMENDORF TH, KOLVRAA S, BISCHOFF C, CHRISTENSEN K, WADSWORTH L, SCHULZER M, LANSDORP PM: Telomere fluorescence measurements in granulocytes and T lymphocyte subsets point to a high turnover of hematopoietic stem cells and memory T cells in early childhood. J Exp Med 190: $157-167,1999$.

SALIQUES S, ZELLER M, LORIN J, LORGIS L, TEYSSIER JR, COTTIN Y, ROCHETTE L, VERGELY C: Telomere length and cardiovascular disease. Arch Cardiovasc Dis 103: 454-459, 2010.

SALPEA KD, NICAUD V, TIRET L, TALMUD PJ, HUMPHRIES SE: The association of telomere length with paternal history of premature myocardial infarction in the European Atherosclerosis Research Study II. $J$ Mol Med (Berl) 86: 815-824, 2008.

SLAGBOOM PE, DROOG S, BOOMSMA DI: Genetic determination of telomere size in humans: a twin study of three age groups. Am J Hum Genet 55: 876-882, 1994.

STRANDBERG TE, STRANDBERG AY, SAIJONMAA O, TILVIS RS, PITKÄLÄ KH, FYHRQUIST F: Association between alcohol consumption in healthy midlife and telomere length in older men. The Helsinki Businessmen Study. Eur J Epidemiol 27: 815-822, 2012.

WILLS LP, SCHNELLMANN RG: Telomere shortening and regenerative capacity after acute kidney injury. $J$ Am Soc Nephrol 21: 202-204, 2010.

WILLS LP, SCHNELLMANN RG: Telomeres and telomerase in renal health. J Am Soc Nephrol 22: 39-41, 2011.

WILSON WR, HERBERT KE, MISTRY Y, STEVENS SE, PATEL, HR, HASTINGS RA, THOMPSON MM, WILLIAMS B: Blood leukocyte telomere DNA content predicts vascular telomere DNA content in humans with and without vascular disease. Eur Heart J 29: 2689-2694, 2008.

WONG LS, VAN DER HARST P, DE BOER RA, HUZEN J, VAN GILST WH, VAN VELDHUISEN DJ: Aging, telomeres and heart failure. Heart Fail Rev 15: 479-486, 2010.

YANG H, FOGO AB: Cell senescence in the aging kidney. J Am Soc Nephrol 21: 1436-1439, 2010.

YOUNG NS: Telomere biology and telomere diseases: implications for practice and research. Hematology Am Soc Hematol Educ Program 2010: 30-35, 2010. 